\title{
Evolución temporal de humedales de la región ayacucho, mediante imágenes modis periodo 2002-2013
}

\section{Temporal evolution of wetlands in the ayacucho region, using modis images, 2002- 2013 period}

\author{
DOI: $10.46932 / \mathrm{sfjdv2n4-076}$
}

Received in: May 1st, 2021

Accepted in: Jun 30th, 2021

\author{
Jorge Luis Lozano Rodriguez \\ Universidad Nacional Agraria La Molina, Perú. \\ Correo electrónico: jorgelozano@lamolina.edu.pe \\ Juan Carlos Chang Chang Fun \\ Universidad Nacional Agraria La Molina, Perú. \\ Correo electrónico: jcchang@lamolina.edu.pe \\ Oscar Enrique Tang Cruz \\ Universidad Nacional Agraria La Molina, Perú. \\ Correo electrónico: otang@ lamolina.edu.pe \\ Eusebio Idelmo Cisneros Tarmeño \\ Universidad Nacional Agraria La Molina, Perú. \\ Correo electrónico: ecisneros@lamolina.edu.pe \\ Hernán Oscar Cortez Gutierrez \\ Universidad Nacional del Callao, Perú. \\ Correo electrónico: hocortez@unac.edu.pe \\ Milton Milciades Cortez Gutierrez \\ Universidad Nacional de Trujillo, Perú. \\ Correo electrónico: mcortezgutierrez@yahoo.es
}

\section{RESUMEN}

La presente investigación responde a la necesidad de caracterizar la evolución temporal de los humedales mediante imágenes satelitales, su procesamiento, análisis, interpretación y discusión, pues los cambios climáticos hacen necesario tener en cuenta que son ecosistemas importantes de gran interés por las diferentes funciones que realizan. Las imágenes satelitales del satélite TERRA del sensor AQUA, fueron procesadas con el software ENVI y se determinó el parámetro del Índice de Vegetación de Diferencia Normalizada (NDVI), más el uso del Google Earth nos permitió a grandes rasgos discriminar entre zonas de vegetación, suelo desnudo, agua y la variación en el tiempo de los Humedales de la Región de Ayacucho. La teledetección nos ofrece grandes progresos en el conocimiento de la naturaleza, aunque es necesario un mayor rigor científico en la interpretación de los resultados y tener como objetivo eliminar los efectos ocasionados por la variabilidad en las condiciones de captación, distorsión provocada por la atmósfera, y la influencia de parámetros radiométricos geométricos tales como la radiancia, reflectancia, emisividad, posición del Sol, pendiente, y altitud. Por lo cual esperamos que contribuya al conocimiento de datos importantes quedando pendiente estimar otros parámetros como la humedad de suelos, evaporación, etc. a través de la cual se precisará y reorientar la tecnología propuesta. 
Palabra claves: Humedales. Teledetección.

\begin{abstract}
This research addresses the need to characterize the temporal evolution of wetlands through satellite images, processing, analysis, interpretation and discussion, as the climate changes make it necessary to consider that are important ecosystems of great interest in the different functions performed. Satellite images of the TERRA satellite AQUA sensor were processed with ENVI software and parameters Index Normalized Difference Vegetation (NDVI) was determined, plus the use of Google Earth allowed us to broadly discriminate between areas of vegetation, soil nude, water and variation in time of Wetlands in the region of Ayacucho. Remote sensing offers great progress in the knowledge of nature, although a greater scientific rigor in the interpretation of the results is necessary and aim to eliminate the effects caused by the variability in the conditions of recruitment, distortion caused by the atmosphere, and the influence of geometric radiometric parameters such as radiance, reflectance, emissivity, position of the Sun, slope, and altitude. So we hope to contribute to the knowledge of important data pending estimate other parameters such as soil moisture, evaporation, etc. through which shall specify and redirect the proposed technology.
\end{abstract}

Keywords: Wetlands. Remote Sensing.

\title{
1 INTRODUCCIÓN
}

Los Humedales constituyen fuentes de aguas superficiales y pueden definirse como cuerpos de agua que llenan las depresiones de la corteza terrestre, siendo el agua, un elemento que está presente en todos los aspectos de la vida, el hombre lo utiliza en todas sus actividades como son las labores domésticas, agrícolas, pecuarias, industriales, recreativas entre otras [1,2,3].

La creación de bases de datos en este tema, reviste importancia decisiva para inventariar y evaluar los recursos hídricos del departamento, con la finalidad de mitigar los efectos de inundación, sequías, desertificación y contaminación [4,5].

La evolución temporal de los humedales de la Región de Ayacucho nos permite comprender como la variación del NDVI de la superficie, son una de las indicadoras de las condiciones del medio en estudio, tales como los estudios medioambientales como hidrológica, geológica, su constitución geológica y por consiguiente, su determinación resulta de gran interés. La determinación de la evolución temporal de los humedales de la Región de Ayacucho a partir de imágenes satelitales realizadas es una tarea complicada debido a la dificultad de la morfología de nuestra región, a un más cuando no se tiene los instrumentos de medición y calculo a la mano por ello se optó en utilizar la ayudad del Google Earth para poder ubicar las coordenadas y direccionar con el programa ENVI $[6,7,8,9]$ la localización de las zonas con NDVI entre - 1 y 0.08 (color rojo) y 0.35 a 1 (color verde) para discriminar zonas con y sin agua. Con lo cual se obtuvo un resultado significativo en la ubicación y la variación en el tiempo de las zonas de humedales de la Región de Ayacucho. 


\section{MATERIALES Y MÉTODOS}

Se ha obtenido y clasificado las imágenes descargadas gratuitamente al suscribirse a un servidor de imágenes del MRTWEB donde se encuentra datos del sensor MODIS, correspondiente al sector de la región de Ayacucho ubicado en el Path (orbita): 05; Row (punto): 69; Latitud: -12 a -13; Longitud: -74 a -73, desde el año 2002 al 2013; todas estas imágenes se procesan mediante el software ENVI 4.8, obteniéndose a partir de ello las imágenes de los lugares direccionados por Google Earth. Para luego determinar la existencia o no de los humedales.

\section{RESULTADOS}

Las imágenes satelitales obtenidas desde el año 2002 al 2013, fueron compiladas y cortadas con el vector Ayacucho para luego proceder a procesar los resultados de las imágenes del índice de vegetación de diferencia normalizada "NDVI" (Normalized Difference Vegetation Index) con el software ENVI y direccionarlas con la ayuda del Google Earth. La localización de las zonas con NDVI entre -1 y 0.08 (color rojo) y $0.35 \mathrm{a} 1$ (color verde), fueron contrastadas con las imágenes de coordenadas obtenidas del Google Earth de fecha 11/27/2013.

Los resultados obtenidos de procesar las imágenes satelitales correspondientes al periodo 2002 al 2013.

Las siguientes graficas muestran los resultados del NDVI desde 2002 al 2013 cuyos valores de las firmas espectrales de los diferentes elementos de las 23 imágenes de todo el año con el cual se puede caracterizar los de índice de vegetación de diferencia normalizada (NDVI) donde los cuerpos de agua se consideran entre -1 y 0.08 que corresponden a la gráfica de rojo.

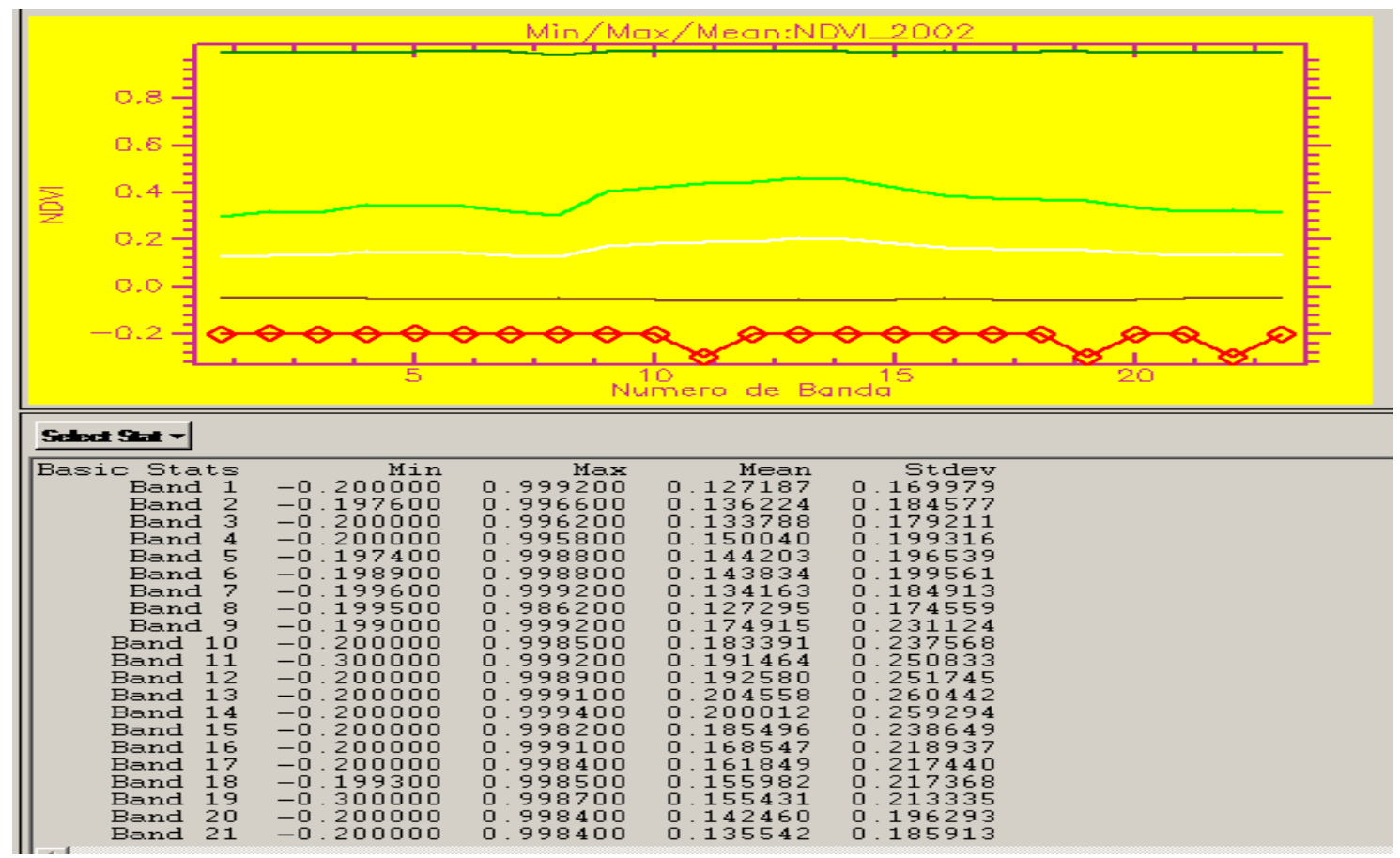



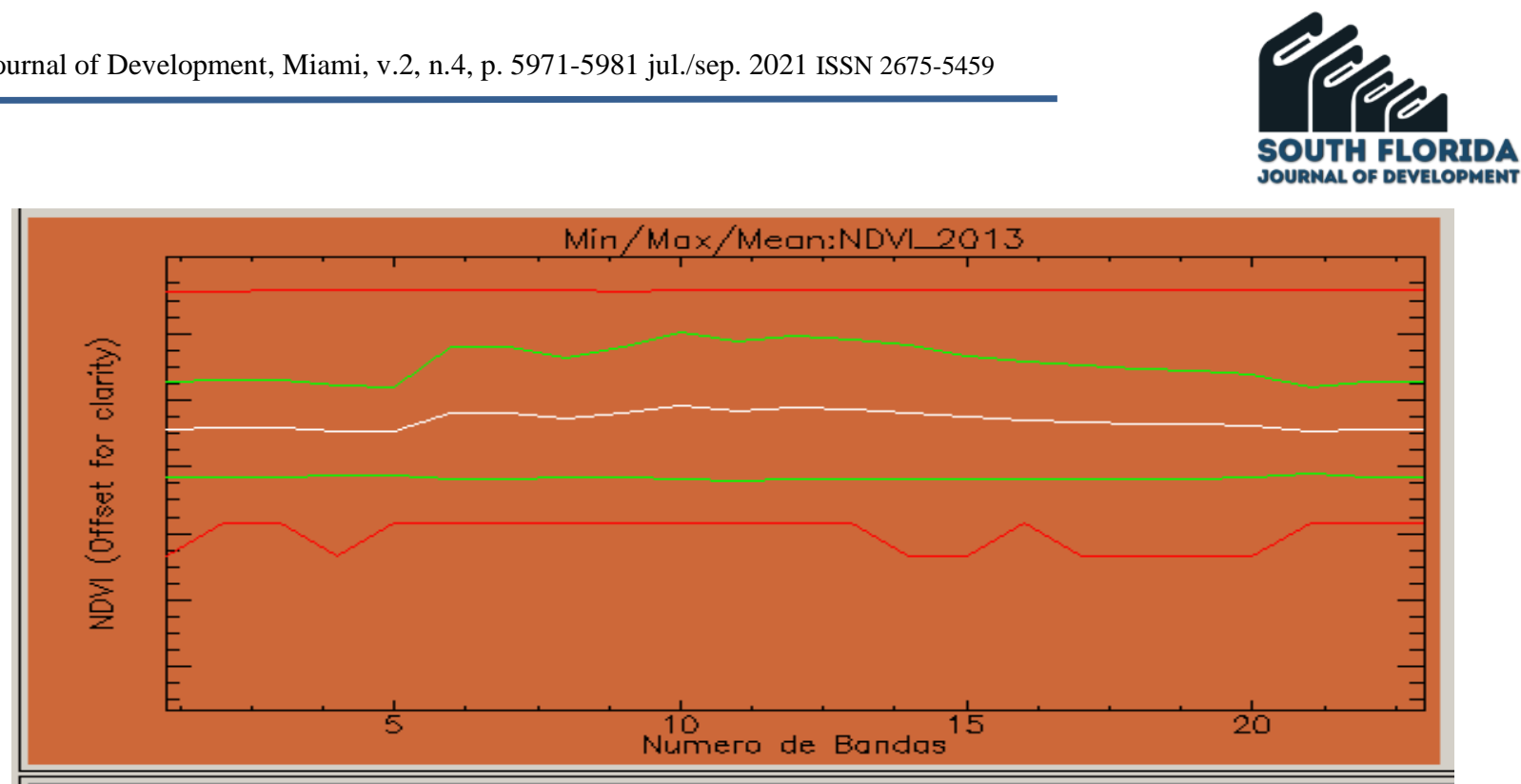

\begin{tabular}{|c|c|c|c|c|}
\hline $\begin{array}{c}\text { Basic Stats } \\
\text { Band } 1 \\
\text { Band } 2 \\
\text { Band } 3 \\
\text { Band } 4 \\
\text { Band } 5 \\
\text { Band } 6 \\
\text { Band } 7 \\
\text { Band } 8 \\
\text { Band } 9 \\
\text { Band } 10 \\
\text { Band } 11 \\
\text { Band } 12 \\
\text { Band 13 } \\
\text { Band } 14 \\
\text { Band } 15 \\
\text { Band } 16 \\
\text { Band 17 } \\
\text { Band 18 } \\
\text { Band 19 } \\
\text { Band } 20 \\
\text { Band } 21 \\
\text { Band } 22\end{array}$ & $\begin{array}{r}\text { Min } \\
-0.300000012 \\
-0.199399993 \\
-0.199200004 \\
-0.300000012 \\
-0.200000003 \\
-0.200000003 \\
-0.199399993 \\
-0.200000003 \\
-0.200000003 \\
-0.200000003 \\
-0.200000003 \\
-0.200000003 \\
-0.200000003 \\
-0.300000012 \\
-0.300000012 \\
-0.200000003 \\
-0.300000012 \\
-0.300000012 \\
-0.300000012 \\
-0.300000012 \\
-0.200000003 \\
-0.200000003\end{array}$ & $\begin{array}{r}\text { Max } \\
0.994899988 \\
0.992100000 \\
0.998199999 \\
0.998700023 \\
0.999300003 \\
0.998600006 \\
0.999599993 \\
0.999300003 \\
0.994300008 \\
0.998700023 \\
0.998700023 \\
0.998300016 \\
0.997799993 \\
0.998899996 \\
0.997399986 \\
0.995599985 \\
0.995400012 \\
0.998799980 \\
0.998199999 \\
0.998399973 \\
0.999199986 \\
0.996399999\end{array}$ & $\begin{array}{l}\text { Mean } \\
0.119083529 \\
0.122391314 \\
0.121114493 \\
0.114669318 \\
0.112081662 \\
0.166394650 \\
0.167665498 \\
0.153679917 \\
0.169422051 \\
0.187595350 \\
0.175584101 \\
0.185418718 \\
0.179182054 \\
0.169373985 \\
0.154795855 \\
0.146318501 \\
0.141443326 \\
0.135898816 \\
0.133213847 \\
0.127212382 \\
0.113813792 \\
0.119196968\end{array}$ & $\begin{array}{l}\text { Stdev } \\
0.167431735 \\
0.172680279 \\
0.170642312 \\
0.160399135 \\
0.158223277 \\
0.223003378 \\
0.223115186 \\
0.204229167 \\
0.221001647 \\
0.245612732 \\
0.234439020 \\
0.241664435 \\
0.234613816 \\
0.226874497 \\
0.208682586 \\
0.200455721 \\
0.197433013 \\
0.191849766 \\
0.187433237 \\
0.179982558 \\
0.155213537 \\
0.166808627\end{array}$ \\
\hline
\end{tabular}

Ubicación geográfica de las 55 lagunas

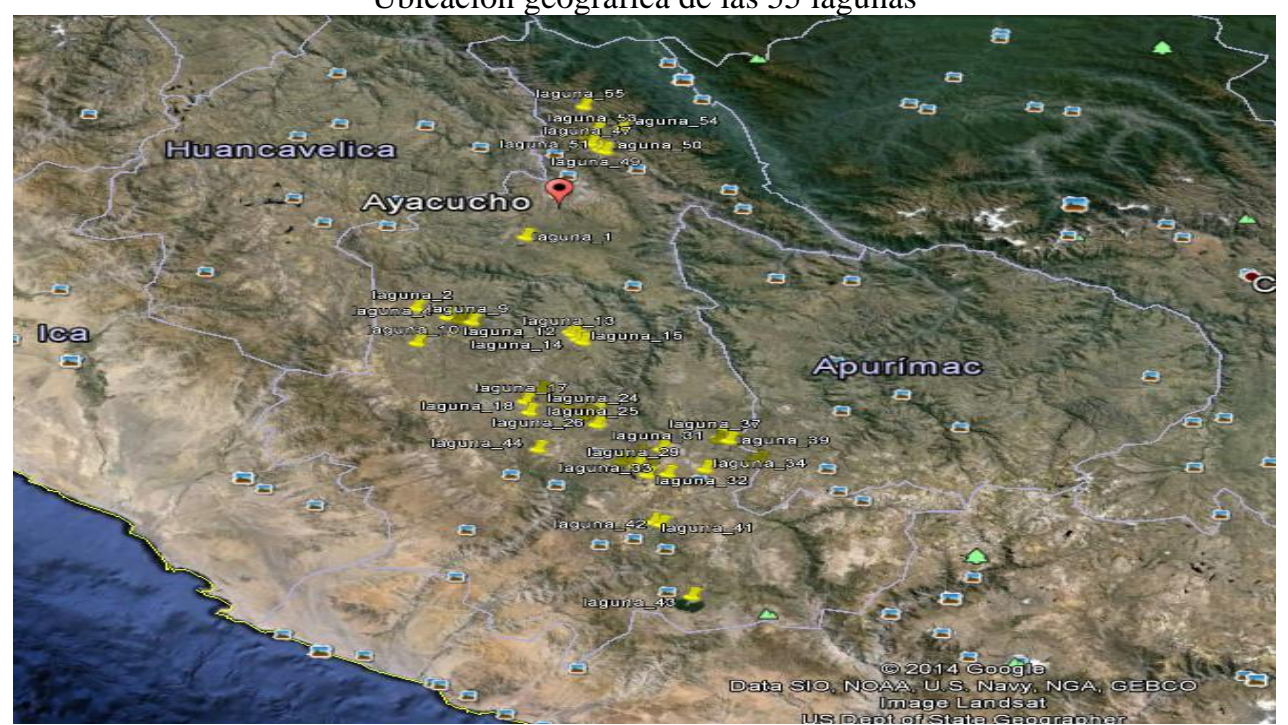

La laguna de latitud $13^{\circ} 26^{\prime} 30.39^{\prime \prime S}$ y Longitud $74^{\circ} 19^{\prime} 48.93 " O$ nos muestra un NDVI Min en la banda 15 (-0.0065) y un NDVI Max en la banda 9 (0.4514). Con el cual se puede clasificar los cuerpos de agua con el software ENVI y Google Earth [10]. 


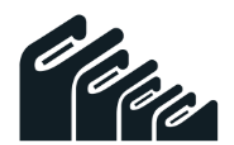 \\ SOUTH FLORIDA \\ JOURNAL OF DEVELOPMENT}

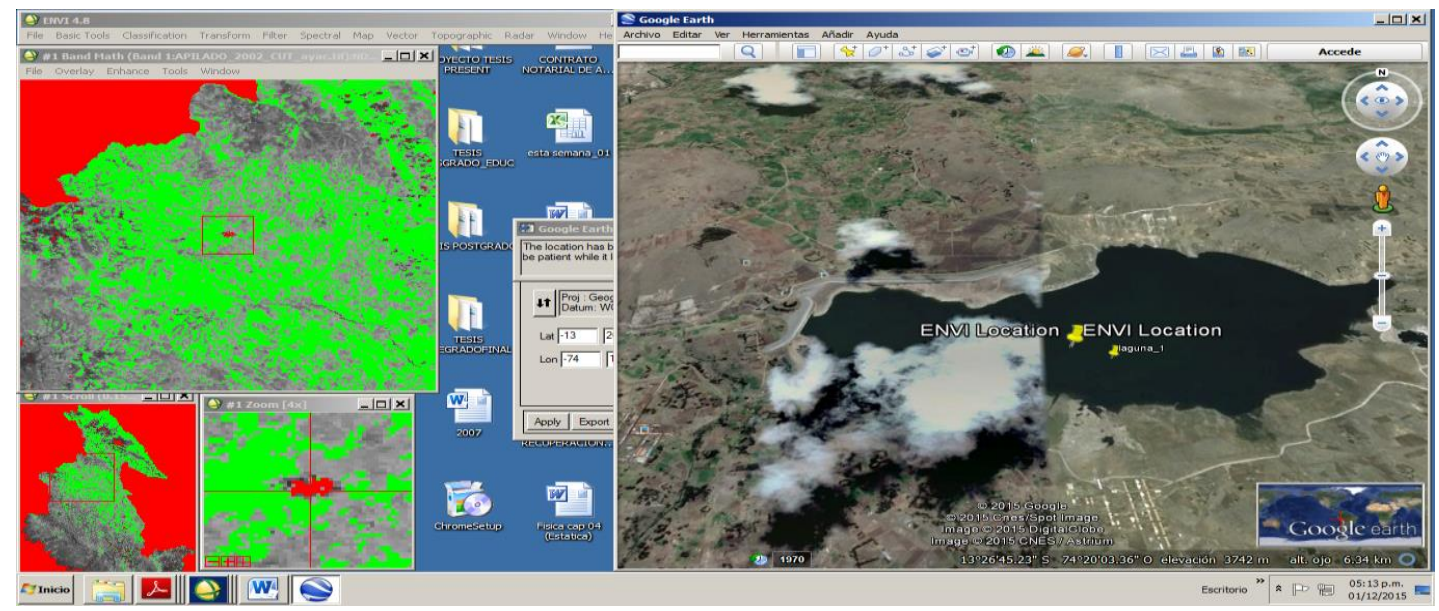

La laguna de latitud $14^{\circ} 36^{\prime} 4.74^{\prime \prime S}$ y Longitud $73^{\circ} 56^{\prime} 10.41^{\prime \prime O}$ nos muestra el sector del cuerpo de agua correlacionando y verificando positivamente con el Google Earth.

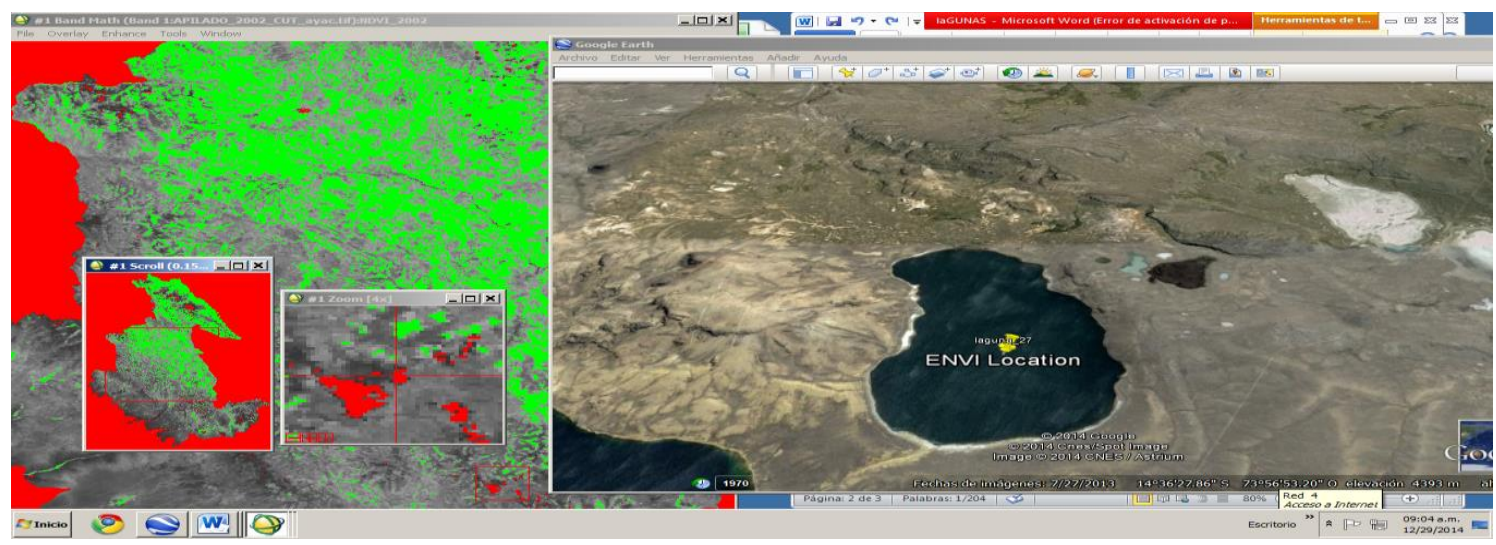

La laguna de latitud $14^{\circ} 38^{\prime} 2.12$ "S y Longitud $73^{\circ} 54^{\prime} 21.67^{\prime \prime O}$ nos muestra el sector del cuerpo de agua correlacionando y verificando positivamente con el Google Earth.

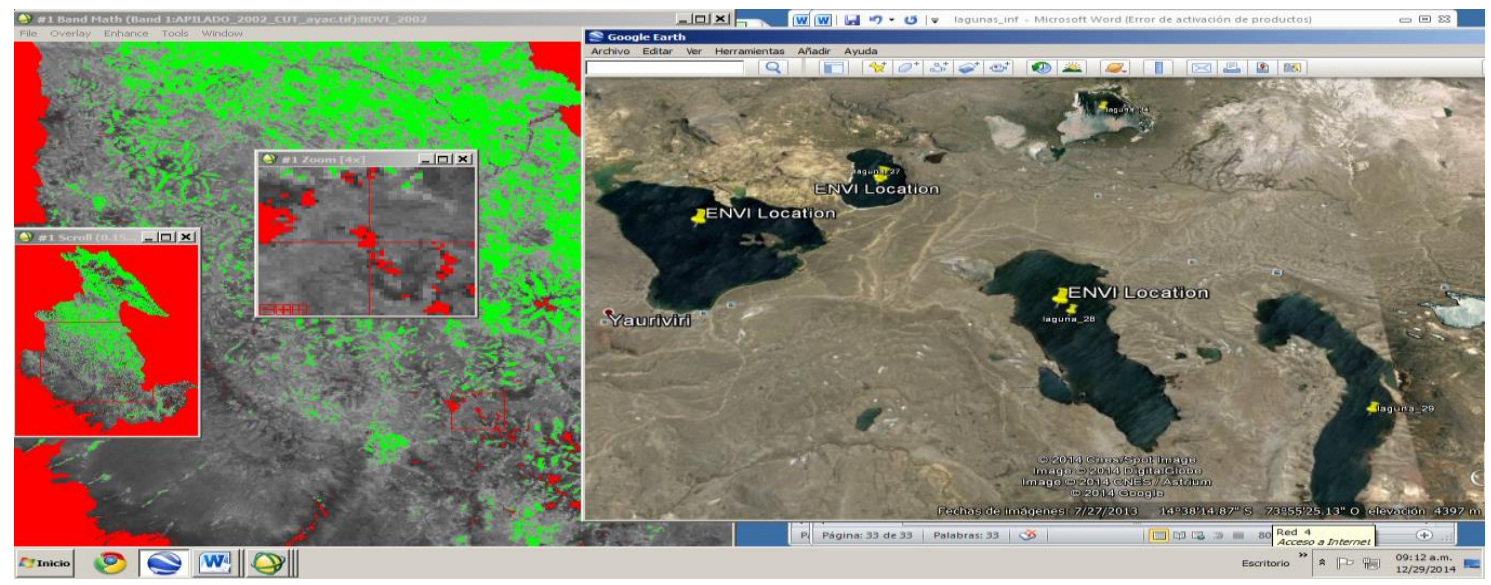

La laguna de latitud $14^{\circ} 40^{\prime} 50.08^{\prime \prime S}$ y Longitud $73^{\circ} 52^{\prime} 18.29^{\prime \prime} \mathrm{O}$ nos muestra el sector del cuerpo de agua correlacionando y verificando positivamente con el Google Earth. 


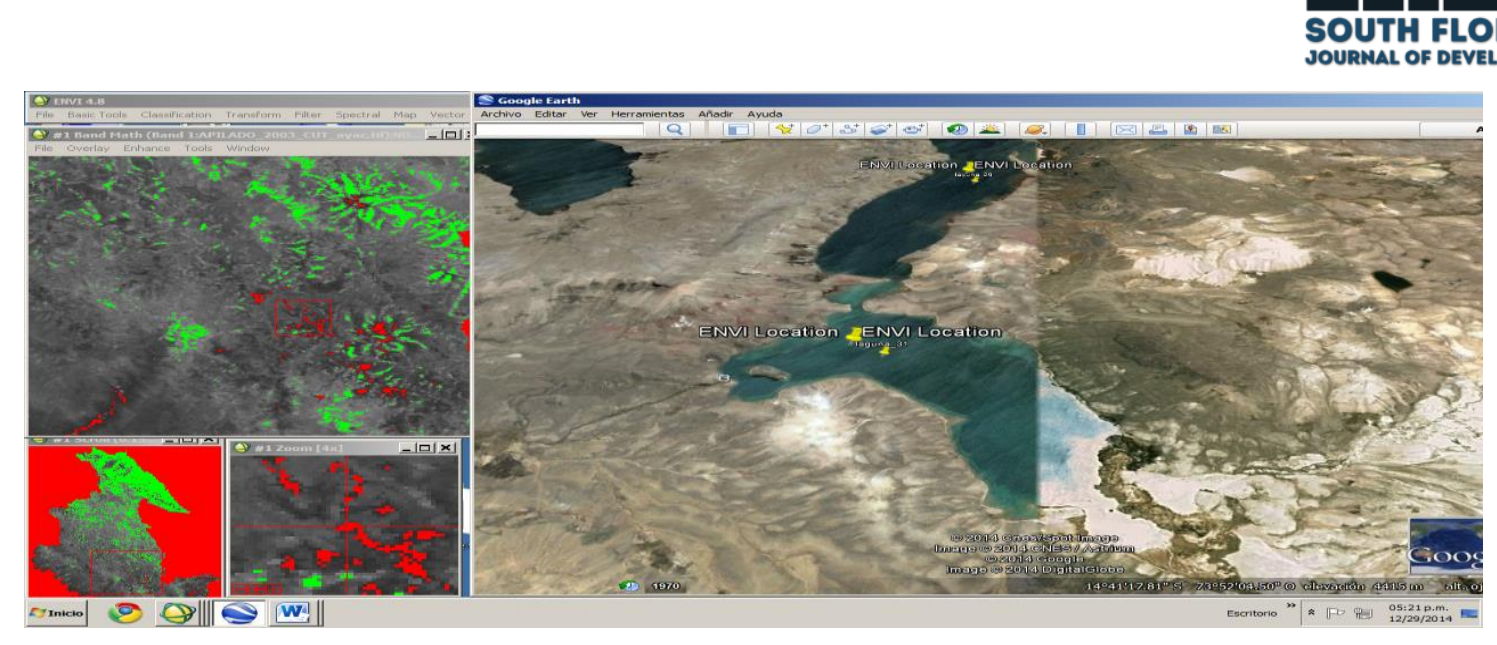

\section{DISCUSIÓN}

1. La información obtenida mediante las imágenes de satélite, confirman la gran diversidad de humedales con comportamientos hídricos muy dispares y diferente estado de conservación, por lo que se ha considerado necesario poner los resultados en una tabla donde se indica las coordenadas y la valoración a cada humedal observada y estudiada.

2. La interpretación de las imágenes dejo resaltar los cambios que se daban en la vegetación entre las tierras altas y bajas, quedando expuesta la asociación entre esta, los tipos de suelos, las alturas y las pendientes

3. Las imágenes satelitales nos permitió determinar que las zonas se encontraban en una transición entre un ecosistema acuático y terrestre, así como también corregir los inconvenientes provocados por los desfasajes de coordenadas entre las imágenes satelitales y el Google Earth.

4. La zonificación realizada a escala de detalle, se obtuvo información sobre la situación de los humedales al comienzo del estudio, asistiendo a una mejor comprensión de su funcionamiento, y se determinó la existencia de zonas más importantes por su extensión se encuentran en las Provincias del Sur del departamento, específicamente en Lucanas y Parinacochas. Con mayor extensión de humedales. En contrapartida se observó que brinda información para un determinado momento que no necesariamente es representativo de la situación habitual de algunos humedales. En este sentido previamente se realizó una exploración con otras fechas y se advirtió que cambiaban bruscamente con el tiempo, llevando a descartar su utilización como mecanismo para zonificar los humedales.

5. Las diferencias en los valores del NDVI, indican la existencia de algún factor que está regulando la productividad de los humedales.

6. La variación temporal de los humedales podría explicarse por el aporte externo de nutrientes, lo cual es uno de los principales elementos que determinan la productividad de un ecosistema de humedales. 
7. La variación temporal y espacial de algunos humedales puede ser establecida a partir de la composición y la situación de la vegetación, vinculada a los cambios espaciales en los regímenes hídricos de los suelos y perturbaciones que sufran las comunidades.

8. La provincia de Huamanga se caracteriza por tener durante la mayor parte de todo el año un clima muy soleado, es decir con gran ausencia de nubes.El clima es templado y seco, con una temperatura promedio de $17.5^{\circ} \mathrm{C}$ y una humedad relativa promedio de $56 \%$. Este clima está considerado como adecuado para la vida y sus principales cultivos son el trigo, maíz y papas. La temporada de lluvias se da entre noviembre y marzo, es pues con tal criterio que se tomó para el procesamiento de las imágenes.

9.La caracterización in situ presentaría un trabajo más exquisito, pero el acceso y el inconveniente de proporcionar una medida puntual, que si bien puede ser suficientemente precisa, no puede tomarse como representativa de zonas extensas. Por esta razón, la única forma de obtener una representación de la ubicación y la variabilidad de los humedales a mayor escala, es mediante el uso de datos suministrados por los sensores ubicados a bordo de plataformas aerotransportadas o satélites artificiales de observación de la Tierra.

\section{CONCLUSIONES}

1. El análisis de los resultados nos genero un nuevo conocimiento sobre el funcionamiento de los humedales considerada apropiada, y concreta, permitiendo dar continuidad a los estudios a lo largo del tiempo, así como también su aplicación a otros ecosistemas y en estudios de diferentes escalas. Se avanzó en establecer el número de humedales, lo que fue posible gracias a las herramientas que representan los SIG y la cartografía preexistente.

2. A partir de la resolución espacial y temporal se desprende que las imágenes MODIS sirven como mecanismos preliminares de exploración, constituyendo un importante insumo que permite hacer análisis de detalle, que en este caso, permitió determinar a grandes rasgos las existencia de grandes zonas de humedales. Como inconvenientes se identifican de no contar con imágenes de alta resolución para su mejor discriminación.

3. Las imágenes MODIS, son una herramienta de gran potencial para el monitoreo y evaluación de la situación de los humedales. En contrapartida se vio que dichas imágenes no aportan una resolución espacial óptima, dado que los pixeles poseen gran tamaño, haciendo que los límites entre zonas sean poco precisos.

4. Para determinar los importantes cambios y alteraciones que suceden en los humedales, se presenta en la actualidad la necesidad de control continuo de sus variables más importantes para conocer la evolución de la salud de los ecosistemas acuáticos. Las premisas para el correcto establecimiento y 
continuidad de un programa de seguimiento deben partir del conocimiento definido del estado óptimo de cada ecosistema en particular.

5. Existen diferentes tipos de variables, tanto físicas como biológicas, que actúan como indicadores del estado de un sistema acuático. Los indicadores fisicoquímicos son los más adecuados para detectar posibles problemas o alteraciones ya que se encuentran íntimamente relacionados con los diferentes procesos que ocurren en el sistema acuático y por ende con los peligros potenciales como la eutrofización, contaminación por vertidos, alteraciones en el régimen del agua, etc. Mientras que los indicadores biológicos o bioindicadores proveen información como la influencia que estos ejercen sobre el ecosistema en el que se desarrollan. 


\section{REFERENCIAS BIBLIOGRÁFICAS}

[1] Ramsar 1996. Resolución VI.12 sobre Inventarios Nacionales de Humedales. Disponible en http://www.ramsar.org/key_res_vi.12.htm.

[2] Ramsar 1999. Resolución VII.20 sobre inventario de humedales. Disponible en http://www.ramsar.org/ key_res_vi.12.htm

[3] Ramsar 2001. 26 Reunión del Comité Permanente de Ramsar - Punto 12.3 (c) (i) del orden del día Marco para el inventario de los humedales. Disponible en http://www.ramsar.org/key_res_vi.12.htm.

[4] Begg GW, van Dam RA, Lowry JB, Finlayson CM \& Walden DJ 2001. Inventory and risk assessment of water dependent ecosystems in the Daly basin, Northern Territory, Australia. Supervising Scientist Report 162, Supervising Scientist, Darwin NT.

[5] Begg GW \& Lowry J 2003. Land capability and topographic data as a surrogate for the mapping and classification of wetlands: a case example from the Daly basin, Northern Territory, Australia.Water Science and Technology, 48(7), 49-56.

[6] DeFries R \& Pagiola S (coordinadores) 2005. Analytical approaches for assessing ecosystem condition and human well-being. En Ecosystems and Human Well-being: Current State and Trends, Evaluación de Ecosistemas del Milenio, World Resources Institute, Washington.

[7] Chuvieco.E: Análisis espectral cartográfica e inventario de tipos de ocupación a partir de imagines Thematic Mapper. “Geographica”. Vol.27 (1985c). pp. 117-129.

[8] ESRI 2004. What is ArcGIS? Disponible en

http://www.esri.com/software/arcgis/about/literature.htm

[9] https://www.yumpu.com/es/document/.../tutorial-de-idl-basico-bosque

Tutorial de IDL básico Salvador Ramirez Flandes. ... Texto fundamental para aprender a programar en $\mathrm{C} / \mathrm{C}++$ - Matematica $\cdot \mathrm{x}$. edu.uy.

[10] M. Bertalmio, L. Vese, G. Sapiro, and S. Osher. Simultaneous structure and textura image inpainting. IEEE Transactions on Pattern Análisis and Machina Intelligence, 12(8):882-889, 2003.

[11] Price, J.C. (1977). Thermal inertia mapping: A new view of the earth. J. Geophys. Res. 82, 25822590.

[12] Short, N.M. y Stuart, L.M. Jr. (1982). The Heat Capacity Mapping Mission (HCMM) anthology, NASA SP-465, NASA Scientific and Technical Information Branch, Washington, D.C.

[13] Price, J.C. (1985). On the Analisys of Thermal Infrared Imagery: The Limited Utility of Apparent Thermal Inertia. Remote Sensing Environment. 18, 59-73.

[14] Utsunomiya, Y. (1996). Construction of a Thermal Inertia Mapping Sistem (TIMS) for Hydrological Analysis of the Earth's Surface using Satellite and Ground Monitory Data. International Archives of Photogrammetry and Remote Sensing, XXXI B, 884-890. 
[15] Tramutoli, V., Claps, P., Marella, M, Pergola, N., Pietrapertosa, C. y Sileo C. (2001). Hidrological implications of remotely sensed termal inertia. Remote Sensing Hydrology. 267, 207-211.

[16] Zhang, R., Sun, X., Zhu, Z., Su, H. y Tang, X. (2003). A remote sensing model for monitoring soil evapor ation based on differential thermal inertia and its validation. Science in China, 46, D, 342-356.

[17] Joshi, D.K. y Panda, B.C. (2007). Simultaneus Measurement of Crop Canopy Temperature and Moisture using Canopy Thermal Inertia. Jour. Agric. Physics, 7, 23-30

[18] Lu, S., Ju, Z. Q., Ren, T. S., y Horton, R. (2009). A general approach to estimate soil water content from thermal inertia. Agricultural and Forest Meteorology, 149, 1693.

[19] Murray, T., y Verhoef, A. (2007). Moving towards a more mechanistic approach in the determination of soil heat flux from remote measurements-I. A universal approach to calculate thermal inertia. Agricultural and Forest Meteorology, 147, 80-87.

[20] Majumdar, T.J., (2003). Regional thermal inertia mapping over the Indian subcontinent using INSAT-1D VHRR data and its possible geological applications. International Journal of Remote Sensing, 24, 2207-2220.

[21] Hardgrove, C., Moersch, J. y Whisner. (2009). Thermal imaging of alluvial fans: A new technique for remote classi?cation of sedimentary features. Earth and Planetary Science Letters. 285, 124-130.

[22] Xue, Y. y Cracknell, A.P. (1995). Advanced thermal inertia modeling. International Journal of Remote Sensing. 16, 431-446.

[23] Sobrino, J.A. y El Kharraz, M.H. (1999). Combining afternoon and morning NOAA sattelites for thermal inertia estimation 1. Algorithm and its testing with hidrologic atmospheric pilot experiment-sabel data, J. Geophys. Res. 104, 9445-9453.

[24] Verhoef, A. 2004. Remote estimation of thermal inertia and soil heat flux for bare soil. Agricultural and Forest and Meteorology, 123, 221-236.

[25] Murray, T. y Verhoef, A. 2007. Moving toward a more mechanistic approach in the determination of soil heat flux from remote measurements, I. A universal approach to calculate thermal inertia. Agricultural and Forest and Meteorology, 147, 80-87.

[26] Murray, T. y Verhoef, A. 2007. Moving toward a more mechanistic approach in the determination of soil heat flux from remote measurements, II. Diurnal shape of soil heat flux. Agricultural and Forest and Meteorology, 147, 88-97.

[27] Van Wijk, W.R. y De Vries, D.A. 1963. Periodic temperature variations in a homogeneous soil. In: Van Wijk, W.R. (Ed.). Physics of Plant Enviroment, North Holland Publishers, Amsterdan, 103-143.

[28] Wang, J., Bras, R.L., Silvandran, G. y Knox, R.G. 2010. A simple method for the estimation of thermal inertia. Geophysical Research Letters, \{\bf 37\}, L055404.

[29] Wang, J. y Bras, R.L. 2009. A model of surface heat fluxes based on the theory of maximum entropy production, Water Resources Research, 45, W03521. 
[30] Nearing, G.S., Moran, M.S. y Scott, R.L. (2012). Coupling diffusion and maximum entropy models to estimate thermal inertia. Remote Sensing of Environment, 119, 222. 